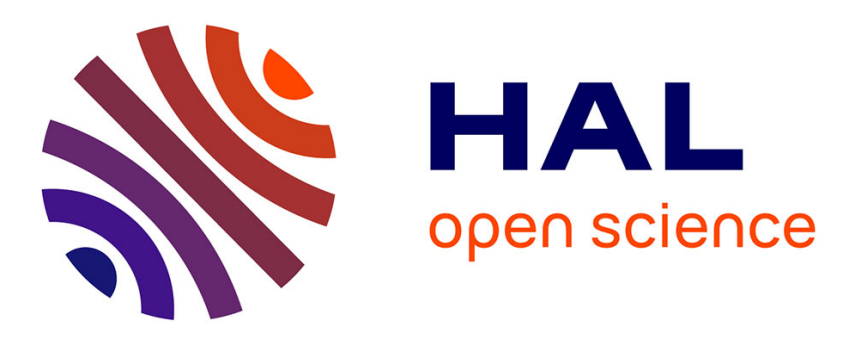

\title{
Simulation of an instrumental childbirth for the training of the forceps extraction: control algorithm and evaluation
}

Richard Moreau, Minh Tu Pham, Xavier Brun, Tanneguy Redarce, Olivier Dupuis

\section{To cite this version:}

Richard Moreau, Minh Tu Pham, Xavier Brun, Tanneguy Redarce, Olivier Dupuis. Simulation of an instrumental childbirth for the training of the forceps extraction: control algorithm and evaluation. IEEE Transactions on Information Technology in Biomedicine, 2011, 15 (3), pp.364-372. 10.1109/TITB.2011.2107746 . hal-00579207

\section{HAL Id: hal-00579207 https://hal.science/hal-00579207}

Submitted on 16 Apr 2019

HAL is a multi-disciplinary open access archive for the deposit and dissemination of scientific research documents, whether they are published or not. The documents may come from teaching and research institutions in France or abroad, or from public or private research centers.
L'archive ouverte pluridisciplinaire HAL, est destinée au dépôt et à la diffusion de documents scientifiques de niveau recherche, publiés ou non, émanant des établissements d'enseignement et de recherche français ou étrangers, des laboratoires publics ou privés.

$$
\text { Copyright }
$$




\title{
Simulation of an instrumental childbirth for the training
}

\section{of the forceps extraction: control algorithm and evaluation}

\author{
R. Moreau*, M. T. Pham*, X. Brun*, T. Redarce* and O. Dupuis ${ }^{\dagger}$ \\ *Laboratoire Ampère, UMR CNRS 5005, Université de Lyon, INSA-Lyon, F-69621 Villeurbanne Cedex, France \\ Email: richard.moreau@insa-lyon.fr \\ ${ }^{\dagger}$ Hospices Civils de Lyon (HCL), Hôpital Lyon-Sud, CHU Lyon Sud, 69495 Pierre-Bénite, France
}

\begin{abstract}
This paper presents the control algorithm implanted on the childbirth simulator BirthSIM in order to provide training to novice obstetricians. The forceps extraction is an obstetric manipulation learned by experience. However, nowadays the training is mainly provided during real childbirths. This kind of training could lead to dramatic consequences due to the lack of experience of some operators. This paper explains the approach which has been used to simulate the dynamic process of a childbirth on the BirthSIM simulator. We especially focus on one procedure which reproduces a difficult instrumental delivery. The recorded tractive force to extract the fetus corresponds to the literature results which confirms the realism of the simulator. The novice results emphasize the need of a childbirth simulator in order to gain initial experience without any risks.
\end{abstract}

Index Terms-Medical robotics, training, position and force control, pneumatic system.

\section{INTRODUCTION}

Novice obstetricians acquire their first obstetric experience directly in the delivery ward. However when complications occur during childbirths, it is difficult to properly learn the correct manipulations. This kind of training is thus not efficient enough to acquire experience and can lead to complications [1]. Apprentice-based training is not efficient or effective for skills acquisition, and inexperience managing complicated childbirth situations can lead to or worsen complications for newborns and mothers.

Complicated childbirths occur when the medical team has to handle obstetric instruments (forceps or vacuum cup) to extract the fetus. Complications of childbirth are quite infrequent, therefore when complications occur it is difficult for novices to gain enough experience to learn and execute surgical gestures and maneuvers effectively. In this paper we focus on the forceps which are the instruments mainly used in the Lyon Hospital Network ("Hospices Civils de Lyon" - HCL). The issue in the forceps use is thus how to learn the correct manipulations without any risk. This leads to a decrease of the forceps use whereas, when they are correctly used, they are the most appropriate instruments i.e. they allow a timely vaginal-assisted childbirth. 
A simulator training allows novice obstetricians to complete their traditional training and offer them the possibility of acquiring an initial experience. Some recent studies provide a solution for the forceps placement training [2]-[4] and evaluation [5]. Once forceps are correctly placed, obstetricians have to proceed to the extraction manipulation. They have to apply the correct amount of forces with their instruments in order to extract the fetus without damaging the fetus and/or the parturient (pregnant woman in the labor phase). The instrumental force produced by obstetricians has to be as small as possible but sufficient to allow the fetus extraction.

The main contribution of this paper is to present and evaluate a control algorithm in order to simulate a difficult instrumental delivery. This paper is divided into four parts. The first part describes the efforts involved during a delivery and the synchronization concepts. The second part is devoted to a brief description of the childbirth simulator BirthSIM [6] and the control algorithm implanted to reproduce the forces is then described. Lastly, the third part concerns the setting of the experimental protocol and the results obtained during the simulation of a difficult forceps extraction are then presented. Finally, the last part discusses these results and presents the future research.

\section{SYNCHRONIZATION OF THE EXPULSIVE FORCES}

\section{A. The maternal expulsive Forces}

The objective of the maternal expulsive forces is to ensure the fetus progression through the maternal pelvis. They have thus to overcome the pelvis muscle resistance which tends to prevent the expulsion. The maternal expulsive forces come from:

- The Uterine Contractions (UC) which are involuntary produced by the parturient at a regular frequency. These UC can be easily identified using a tocography which provides the intra-abdominal pressure variation as a function of time. The UC are the source of an Involuntary Expulsive Force (IEF) [7].

- The abdominal pressure the parturient exerts on her uterus which leads to produce a Voluntary Expulsive Force (VEF). This force is voluntarily produced by the parturient, and its aim is to complete the IEF in order to overcome the natural resistive force due to the pelvic muscles.

Concerning the values of these forces, approximations can be found in the literature [7], [8]. During the expulsion phase of the delivery, the maximum total intensity of an UC can reach $9.7 \mathrm{kPa}$. Applied on a surface around $85 \times 10^{-4} \mathrm{~m}^{2}$ a 9.7 $\mathrm{kPa}$ UC produces an IEF amplitude around $82 \mathrm{~N}$. The VEF intensity is bonded to the parturient health and its intensity can reach $200 \mathrm{~N}$.

A Gaussian function is the simplest model to simulate the shape of the maternal expulsive force. For the IEF, this function is $P$ periodic to ensure the simulation of several regular UC. The Gaussian function, denoted by IEF(t), used is defined by:

$$
I E F(t)=I\left(\exp \left(-\frac{t^{2}}{d}\right)\right)+B_{t}
$$

with 
- $t \in\left[t_{1}, t_{2}\right], t_{1}$ and $t_{2} \in \mathbb{R}$ determine the period $P=\left(t_{2}-t_{1}\right)$.

- $d \in \mathbb{R}^{+}$corresponds to the IEF duration and allows to tune the Gaussian function width. The function is considered as null when it is below a threshold of 0.05 (arbitrary chosen value).

- $I \in \mathbb{R}^{+}$defines the IEF true intensity. As the function $\exp \left(-\frac{t^{2}}{d}\right)$ varies between 0 and $1, I$ tunes the function amplitude (between 60 and $100 \mathrm{~N}$ ) [8].

- $B_{t} \in \mathbb{R}^{+}$is the basic tone of the UC. It corresponds to the minimum value between two UC.

Fig. 1 shows a simulated IEF with:

- a 180 second period;

- a 60 second duration;

- a $70 \mathrm{~N}$ true intensity;

- a $10 \mathrm{~N}$ basic tone.

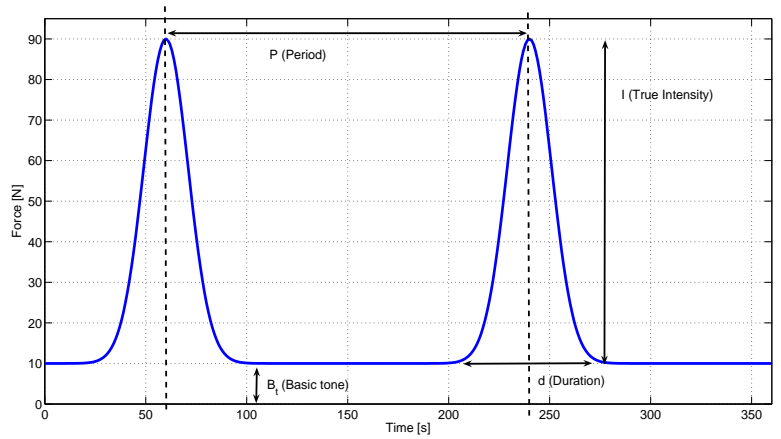

Figure 1. The different parameters of an IEF

Concerning the VEF a similar function, denoted $\operatorname{VEF}(\mathrm{t})$, is used:

$$
V E F(t)=I_{V E F}\left(\exp \left(-\frac{t^{2}}{d_{V E F}}\right)\right)
$$

with

- $t \in\left[t_{3}, t_{4}\right], t_{3}$ and $t_{4} \in \mathbb{R}$ are pre-determined but can be changed by the instructor, their values correspond to a 50 second duration. This function is not periodic because it can be triggered by the instructor on the operator demand.

- $d_{V E F} \in \mathbb{R}^{+}$, is the Gaussian function width;

- $I_{V E F} \in \mathbb{R}^{+}$tunes the intensity of the VEF. It can vary from 0 to $200 \mathrm{~N}$.

\section{B. The Instrumental Tractive Force (ITF)}

For some deliveries the IEF and VEF are unfortunately not sufficient to allow the fetus progression. That is why the obstetricians have to add an external force to help the fetus progression. This force, called Instrumental Tractive Force (ITF), is applied by the obstetricians with their obstetric instruments which could be a vacuum cup or forceps. The ITF aim is to complete the IEF and the VEF to overcome the resistive force of the pelvic muscle.

It is noteworthy that there are not any accurate values in the literature for the ITF due to the difficulty of carrying out in vivo measurements. Nevertheless some researchers have tried to quantify this force by instrumenting some forceps with force sensors. The results obtained are not very conclusive and vary between $150 \mathbf{N}$ and $300 \mathbf{N}$ according to the instrumentation used (dynanometer [9], strain gauges [10], [11], water-inflatable sensors [12], theoretical calculations based on the maximum pressure of the amniotic liquid [13]). More details are available in [14]. One contribution of our work is to provide a realistic estimation of the ITF with forceps thanks to a childbirth simulator. 
C. Synchronization of the expulsive forces

To obtain successful instrumental deliveries, the sum of the expulsive forces, denoted Total Expulsive Force (TEF), has to be superior to the resistive force. This principle leads to the concepts of simple and double synchronization. Its aim is to optimize the TEF to ensure the fetus progression with a minimum ITF in order to obtain an instrumental delivery as close as an eutocic delivery (when obstetric instruments are not necessary). When only two expulsive forces are involved (IEF and VEF or IEF and ITF), we talk about simple synchronization concept. The double synchronization concept appears when the three expulsive forces are involved as it is often the case during instrumental deliveries. Fig. 2 shows an example of a simple and an excellent double synchronization.

On these figures, the resistive force due to pelvic muscles is arbitrary fixed around 200 N. This value can change according to the delivery difficulty.
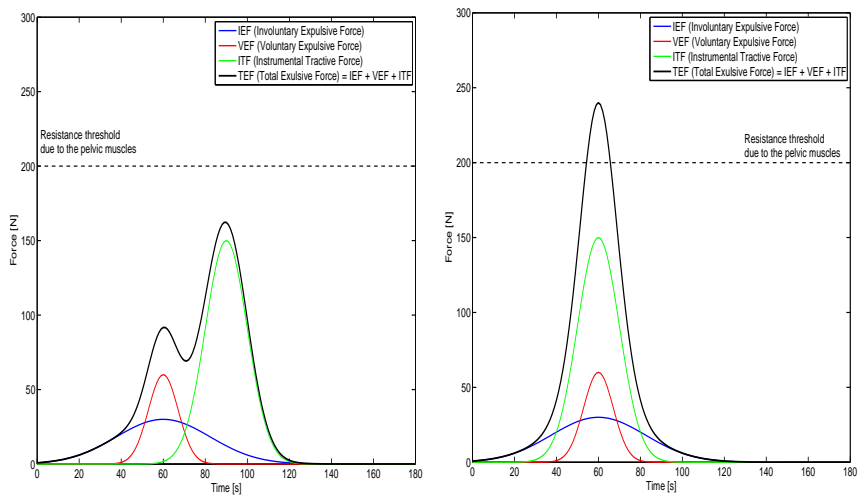

(a) Simple synchronization of the IEF (b) Excellent synchronization: all exand the VEF but ITF is applied too pulsive forces are synchronized late

Figure 2. Simple and double synchronization concepts

\section{Simulation OF THE FORCES ON THE CHILDBIRTH}

\section{SIMULATOR BIRTHSIM}

\section{A. The childbirth simulator BirthSIM}

The BirthSIM simulator is composed of [6]:

- A mechanical component to ensure the anthropomorphism of the simulator (a pelvis and a fetal head manikins with their respective anatomical landmarks). It allows obstetricians to have haptic sensations.

- An electro-pneumatic component to reproduce the dynamic process of a delivery. It consists of a pneumatic actuator with a servodistributor to simulate the different efforts involved during delivery.

- A visualization interface to offer complementary data to operators such as the forceps positions inside the maternal pelvis or information on the current simulated delivery procedure.

\section{B. The electro-pneumatic component}

Fig. 3 shows the principle diagram of the electro-pneumatic component of the BirthSIM simulator, which consists of:

- a pneumatic actuator;

- a servodistributor $5 / 3$ for the mass flow rate regulation;

- two pressure sensors mounted in each chamber of the pneumatic actuator;

- a potentiometer sensor to measure the position of the fetal head;

- a force sensor to measure the traction and compression forces applied on the fetal head. It is mounted between this last one and the pneumatic actuator extremity. 


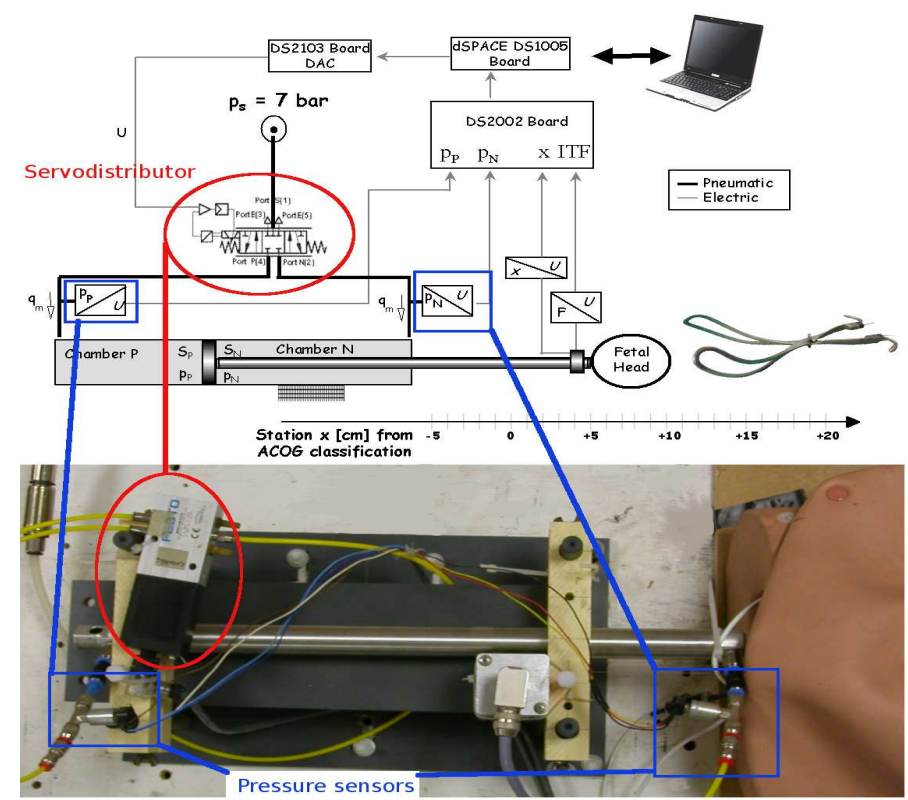

Figure 3. Principle diagram of the electro-pneumatic component of the BirthSIM simulator

Concerning the position of the fetal head, it corresponds to its position with respect to the ischial spines plane as defined by the ACOG (American College of Obstetrics and Gynecology) classification [15], [16]. It is called station, and station 0 means that the top of the head is tangent to the transverse plane gathering the ischial spines.

The source pressure $p_{s}$ equals to 7 absolute bar. With such a source pressure, the pneumatic actuator is able to reproduce the different forces highlighted in subsection II-A. In order to simulate different kinds of childbirth, several procedures are available on the BirthSIM simulator. They are briefly presented without any experimental results in [17].

The electropneumatic system model can be obtained using three physical laws: the mass flow rate through a restriction, the pressure behaviour in a chamber with variable volume and the fundamental mechanical equation. Tab I sums up the main symbols and their description used in this paper.
Table I

NOMENCLATURE

\begin{tabular}{|c|c|c|}
\hline Symbol & Description & Units \\
\hline$b_{v}$ & Viscous friction coefficient & {$[\mathrm{N} / \mathrm{m} / \mathrm{s}]$} \\
\hline$C_{p}^{e}$ & $\begin{array}{l}p \text { partial derivative of the mass flow rate } \\
\text { around equilibrium state }\end{array}$ & {$[\mathrm{kg} / \mathrm{s} / \mathrm{Pa}]$} \\
\hline$\delta X$ & Small variation of $\mathrm{X}$ around an equilibrium point & \\
\hline$F_{\text {ext }}$ & External force & {$[\mathrm{N}]$} \\
\hline$F_{f}$ & Friction force & {$[\mathrm{N}]$} \\
\hline$F_{p r}$ & Pneumatic force & {$[\mathrm{N}]$} \\
\hline$F_{s}$ & Stiction force & {$[\mathrm{N}]$} \\
\hline$G_{u}^{e}$ & $\begin{array}{l}u \text { partial derivative of the mass flow rate } \\
\text { around equilibrium state }\end{array}$ & {$[\mathrm{kg} / \mathrm{s} / \mathrm{V}]$} \\
\hline$k$ & Polytropic constant & \\
\hline$M$ & Total moving load mass & {$[\mathrm{kg}]$} \\
\hline$p_{X}$ & Pressure in the chamber $\mathrm{X}$ & {$[\mathrm{Pa}]$} \\
\hline$q_{m}$ & Mass flow rate & {$[\mathrm{kg} / \mathrm{s}]$} \\
\hline$r$ & Perfect gas constant & {$[\mathrm{J} / \mathrm{kg} / \mathrm{K}]$} \\
\hline$S_{P}, S_{N}$ & Piston areas & {$\left[\mathrm{m}^{2}\right]$} \\
\hline$T$ & Ambient temperature & {$[\mathrm{K}]$} \\
\hline$u_{P}, u_{N}$ & Servodistributor voltages & {$[\mathrm{V}]$} \\
\hline$V_{D X}$ & Dead volume of chamber $\mathrm{X}$ & {$\left[\mathrm{m}^{3}\right]$} \\
\hline$V_{X}$ & Volume of chamber X & {$\left[\mathrm{m}^{3}\right]$} \\
\hline$v$ & Velocity & {$[\mathrm{m} / \mathrm{s}]$} \\
\hline$x$ & Position & {$[\mathrm{m}]$} \\
\hline
\end{tabular}

Using classical assumptions [18], [19] a nonlinear model of the study process [20] can be obtained. Around an equilibrium set (denoted with the exponent $e$ ), a tangent linearized model can be established from this nonlinear model [21]: 


$$
\frac{d}{d t}\left[\begin{array}{c}
\delta p_{P} \\
\delta p_{N} \\
\delta v \\
\delta x
\end{array}\right]=\left[\begin{array}{cccc}
-\frac{1}{\tau_{P}^{e}} & 0 & -\frac{k p_{P}^{e} S_{P}}{V_{P}\left(x^{e}\right)} & 0 \\
0 & -\frac{1}{\tau_{N}^{e}} & \frac{k p_{N}^{e} S_{N}}{V_{N}\left(x^{e}\right)} & 0 \\
\frac{S_{P}}{M} & -\frac{S_{N}}{M} & -\frac{b_{v}}{M} & 0 \\
0 & 0 & 1 & 0
\end{array}\right]\left[\begin{array}{c}
\delta p_{P} \\
\delta p_{N} \\
\delta v
\end{array}\right]+\left[\begin{array}{c}
\frac{k r T}{V_{P}\left(x^{e}\right)} G_{u_{P}}^{e} \\
-\frac{k r T}{V_{N}\left(x^{e}\right)} G_{u_{N}}^{e} \\
0 \\
0
\end{array}\right] \delta u
$$

With the time constants $\tau_{P}^{e}$ and $\tau_{N}^{e}$ defined by:

$$
\tau_{P}^{e}=\frac{V_{P}\left(x^{e}\right)}{k r T C_{p_{P}}^{e}} \text { and } \tau_{N}^{e}=\frac{V_{N}\left(x^{e}\right)}{k r T C_{p_{N}}^{e}}
$$

Where

$$
\left\{\begin{array} { l } 
{ V _ { P } ( x ) = V _ { P } ( 0 ) + S _ { P } x } \\
{ V _ { N } ( x ) = V _ { N } ( 0 ) - S _ { N } x }
\end{array} \text { with } \left\{\begin{array}{l}
V_{P}(0)=V_{D P}+S_{P} \frac{\text { Stroke }}{2} \\
V_{N}(0)=V_{D N}-S_{N} \frac{\text { Stroke }}{2}
\end{array}\right.\right.
$$

And the mass flow rate sensibility coefficients with respect to the pressures $p$ and the control $u$ are deduced from the nonlinear global static characteristic of the FESTO servodistributor [22]:

$$
\left\{\begin{array}{c}
C_{p_{P}}^{e}=-\left.\frac{\partial q_{m}\left(u^{e}, p_{P}\right)}{\partial p_{P}}\right|_{e}, \quad G_{u_{P}}^{e}=\left.\frac{\partial q_{m}\left(u, p_{P}^{e}\right)}{\partial u}\right|_{e} \\
C_{p_{N}}^{e}=\left.\frac{\partial q_{m}\left(u^{e}, p_{N}\right)}{\partial p_{N}}\right|_{e}, \quad G_{u_{N}}^{e}=-\left.\frac{\partial q_{m}\left(u, p_{N}^{e}\right)}{\partial u}\right|_{e}
\end{array}\right.
$$

\section{Control algorithm to simulate instrumental deliveries}

To simulate instrumental deliveries, the first idea was to use a force tracking control. As explained in section II, involved forces can be divided into two kinds: the resistive force due to the pelvic muscle and the expulsive force stemming from the parturient. Our idea was to use the mechanical friction force of our system to reproduce the resistive force and the force stemmed from the pneumatic actuator to reproduce the parturient forces. Unfortunately, the friction forces on our system are not sufficient to be realistic. We therefore decided to choose another control algorithm which is presented in this section.

During the simulation of instrumental deliveries, obstetricians have to apply the correct amounts of force to extract the fetus in order to minimize the risks linked to their manipulations. In this paper we will mainly focus on the procedure which reproduces a difficult forceps extraction. It simulates the case where the parturient forces (the IEF and the VEF) are not sufficient to ensure the fetus progression, so the operator has to use forceps to extract the fetus.

The aim of the control algorithm is to reproduce all the forces involved during an instrumental delivery: the IEF, the VEF, but also the resistive force which tends to prevent the fetus progression. As previously said, the mechanical frictions are not sufficient. To overcome this problem another simple but efficient solution was chosen: the use of a position feedback control with a sliding position gain to control the stiffness of the pneumatic actuator. This technique permits to reduce cost and design complexity of our system. The implemented control law is thus:

$$
U=K_{x}\left(x_{d}-x\right)
$$

And the equivalent control scheme is shown on Fig. 4.

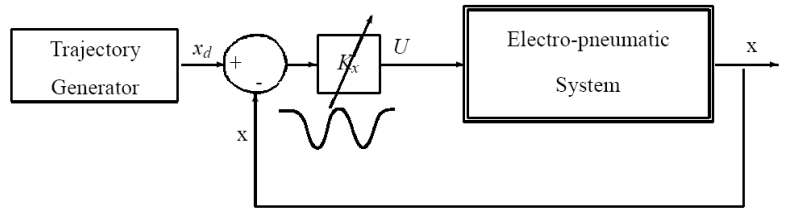

Figure 4. Position feedback control

The objectives of this algorithm is to reproduce realistic haptic sensations and in particular to substitute the role of the 
expulsive parturient forces. The particularity of this control law lies on:

- $K_{x}$ is not a constant but it is a time varying parameter;

- $x_{d}$ is a desired position provided by a trajectory generator depending on the position of the fetal head.

\section{1) The trajectory generator:}

During real deliveries the fetal head progresses by step and can be submitted to back and forth displacements if the obstetrician does not apply sufficient forces to maintain it at its current position. To simulate realistic deliveries a trajectory generator is implemented according to this algorithm:

1. $x_{d}=x_{\text {init }}$

2. while $x<x_{\text {end }}$ do

3. if $x-x_{d}>$ step

$$
x_{d}=x_{d}+\text { step }
$$

\section{endif}

\section{4. endwhile}

$x_{d}$ and $x_{\text {init }}$ are respectively the desired and the initial position of the fetal head. The extraction is considered as completed when the final position, $x_{\text {end }}$, is reached. step is the value of the position increment to validate a displacement inside the maternal pelvis. All these values are chosen before the experiments by the experienced obstetrician who plays the key role of an instructor. $x_{d}$ is thus incremented of the step value if the operator manages to displace the head of at least the step value. An example of the behavior of the desired position $x_{d}$ is shown on Fig. 5. Typical values for all these parameters are $x_{\text {init }}=2 \mathrm{~cm}, x_{\text {end }}=15 \mathrm{~cm}$, and step $=1 \mathrm{~cm}$.

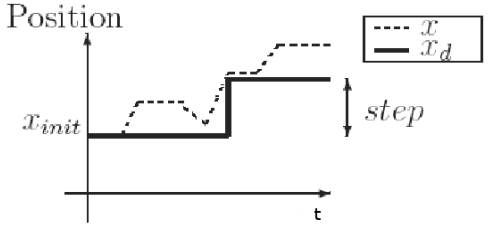

Figure 5. $x_{d}$ behavior due to the trajectory generator

2) Tuning of the stiffness of the pneumatic actuator:

The applied ITF to move the fetal head depends on the moment where the operator exerts it (concepts of synchronization). To reproduce this behavior, we chose to regulate the stiffness $K$ of the pneumatic actuator. The stiffness of the pneumatic actuator is linked to the actuator force $F$ and to the displacement $x$ by:

$$
K=\frac{\partial(\delta F)}{\partial(\delta x)}
$$

Where the actuator force $F$ is:

$$
F=F_{p r}-F_{f}(v)-F_{e x t}
$$

And:

- $F_{p r}=S_{P} p_{P}-S_{N} p_{N}$ is the pneumatic force.

- $F_{f}(v)=b_{v} v+F_{s} \operatorname{sgn}(v)$ is the friction force with $\operatorname{sgn}(v)$ depends on the sign of $v:-1$ if $v<0$ and 1 if $v>0$;

- $F_{\text {ext }}=\left(S_{P}-S_{N}\right) p_{\text {atm }}$ is the force due to the atmospheric pressure $p_{\text {atm }}$

Considering the model given by equation (3) and assuming that the dynamic of the pressures in the chambers (more or less one second) can be neglected in regards of the dynamic of the UC (about 60 seconds) the relation between pressure and control is given by:

$$
\left\{\begin{array}{l}
\delta p_{P}=\frac{G_{u_{P}}^{e}}{C_{p_{P}}^{e}} \delta u-\frac{p_{P}^{e} S_{P}}{r T C_{p_{P}}^{e}} \delta v \\
\delta p_{N}=-\frac{G_{u_{N}}^{e}}{C_{p_{N}}^{e}} \delta u+\frac{p_{N}^{e} S_{N}}{r T C_{p_{N}}^{e}} \delta v
\end{array}\right.
$$

It corresponds to the pressure gains of the servodistributor around the equilibrium set. For this application the variations 
of the pressures and control are very small, about 0.2 bar and $20 \mathrm{mV}$ respectively (see experimental results IV-A), so the pressure gain can be considered as constant. The pneumatic force variations are thus:

$$
\begin{aligned}
& \delta F_{p r}=S_{P} \delta p_{P}-S_{N} \delta p_{N} \\
& \delta F_{p r}=\left(S_{P} \frac{G_{u_{P}}^{e}}{C_{p_{P}}^{e}}+S_{N} \frac{G_{u_{N}}^{e}}{C_{p_{N}}^{e}}\right) \delta u-\left(\frac{p_{N}^{e} S_{N}^{2}}{r T C_{P_{N}}^{e}}+\frac{p_{P}^{e} S_{P}^{2}}{r T C_{p_{P}}^{e}}\right) \delta v
\end{aligned}
$$

The external force, $F_{\text {ext }}$, is constant and the variation of the friction force is:

$$
\delta F_{f}(v)=b_{v} \delta v+\delta\left(F_{s} \operatorname{sgn}(v)\right)
$$

Once the fetal head is extracted by the operator, it moves out the maternal pelvis and so $v$ has always the same sign and as $F_{S}$ is a constant, the variation $\delta\left(F_{S} \operatorname{sgn}(v)\right)$ is therefore null. Concerning non smooth trajectories when the fetal is in movement, $\operatorname{sgn}(v)$ changes but there is no more the stick effect i.e. $F_{s}=0$ and thus $\delta\left(F_{s} \operatorname{sgn}(v)\right)$ is null.

Thus from (9), (11), and (12), we can write:

$$
\begin{aligned}
& \delta F=\delta F_{p r}-\delta F_{f}(v) \\
& \delta F=\left(S_{P} \frac{G_{u_{P}}^{e}}{C_{p_{P}}^{e}}+S_{N} \frac{G_{u_{N}}^{e}}{C_{p_{N}}^{e}}\right) \delta u-\left(\frac{p_{N}^{e} S_{N}^{2}}{r T C_{p_{N}}^{e}}+\frac{p_{P}^{e} S_{P}^{2}}{r T C_{p_{P}}^{e}}+b_{v}\right) \delta v
\end{aligned}
$$

Using a control law with feedback in position with scheduled gain given by equation (7) and for a constant desired value, the relation between force and position is:

$$
\delta F=-\left(S_{P} \frac{G_{u_{P}}^{e}}{C_{p_{P}}^{e}}+S_{N} \frac{G_{u_{N}}^{e}}{C_{p_{N}}^{e}}\right) K_{x} \delta x-\left(\frac{p_{N}^{e} S_{N}^{2}}{r T C_{p_{N}}^{e}}+\frac{p_{P}^{e} S_{P}^{2}}{r T C_{p_{P}}^{e}}+b_{v}\right) \delta v
$$

So we can identify the stiffness and the viscous coefficients in closed loop, respectively denoted $K$ and $B$ :

$$
\begin{aligned}
& \delta F=-K \delta x-B \delta v \\
& K=\left(S_{P} \frac{G_{u_{P}}^{e}}{C_{p_{P}}^{e}}+S_{N} \frac{G_{u_{N}}^{e}}{C_{p_{N}}^{e}}\right) K_{x} \\
& B=\left(\frac{p_{N}^{e} S_{N}^{2}}{r T C_{p_{N}}^{e}}+\frac{p_{P}^{e} S_{P}^{2}}{r T C_{p_{P}}^{e}}+b_{v}\right)
\end{aligned}
$$

So the stiffness variation of our system in closed loop is guaranteed by the variation of the controller gain $K_{x}$. Indeed stiffness variations are considered as a substitute to the variation of the parturient forces i.e. when the maternal expulsive forces increase, the stiffness of the system has to decrease, and conversely when no maternal expulsive forces are present, the stiffness value should be high. The variation of the force is opposite to the variation of the position because when the fetal head is extracted $(\delta x>0)$ the force variation tend to maintain the head inside the pelvis $(\delta F<0)$.

One difficulty in the synthesis of the control law is the tuning of the different gains because only the experience of the obstetrician can provide a realistic haptic feedback of an instrumental delivery.

The parameter values of the exponential function presented in (1) and (2) are thus defined empirically with an expert obstetrician. We asked him to exert an ITF as though he was in a delivery while the IEF is absent and when it is rising. This allows to determine the amplitude of the gaussian function according to his clinical experience in order to let him have realistic haptic sensations. Concerning the duration parameter it has the same value than the IEF and the VEF.

\section{EXPERIMENTAL RESULTS}

\section{A. Validation of the model}

To validate the hypothesis presented in the previous section (small variations of control and pressures), we implemented the control law (7) on the system. Fig. 6 shows the control signal $U$ applied to the system, the measured $p_{P}$ and $p_{N}$ for a given $K_{x}$. 

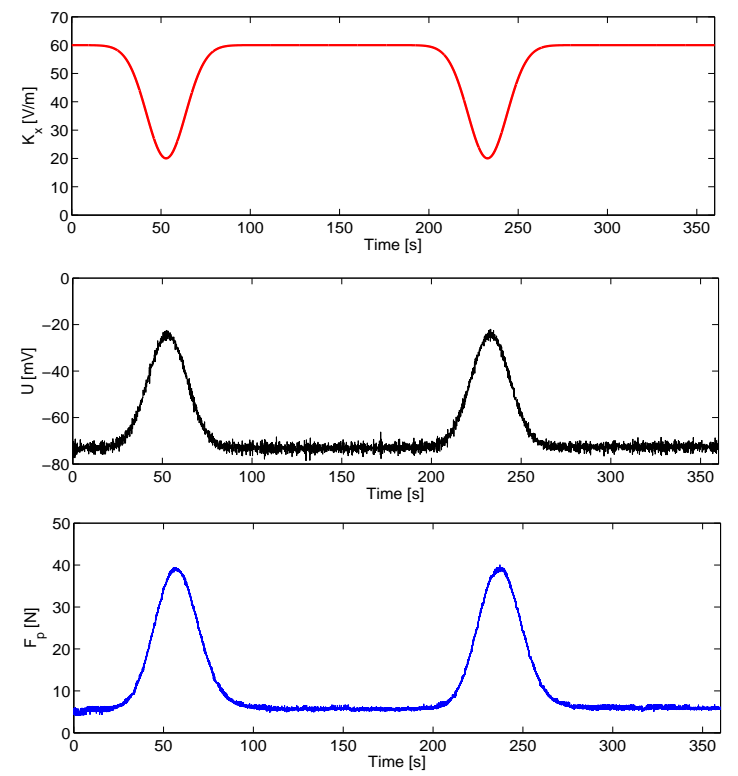

Figure 6. For a given $K_{x}$ the obtained $U$ and $F_{P}$ to simulate a difficult forceps extraction

Due to the trajectory generator implemented (Fig. 5), $x$ is always superior or equal to $x_{d}$. So, from (7) we can conclude that $x_{d}-x$ is always negative and thus the control $U$ behaves in the opposite way of $K_{x}$ as shown on Fig. 6.

\section{B. Experimental Protocol}

The main goal of the experiment is to offer obstetricians the opportunity to handle a risk free forceps extraction. It allows them to be aware of the forces involved during a difficult instrumental delivery. The objective is to show them the synchronization concepts. Novices are junior obstetricians with less than twelve months of obstetrical experience. During all the experiments, only the forceps extraction manipulation is studied.

The initial conditions of all attempts are:

- The fetal head is placed in $\mathrm{OA}+2$. OA means OccipitoAnterior location: forceps have to be placed in a symmet- rical way. +2 means that the fetal head is at $2 \mathrm{~cm}$ from the ischial spines plane: forceps are thus placed deep inside the maternal pelvis.

- IEF is simulated by a stiffness variation of the pneumatic actuator. The duration is 60 seconds and the periodicity is every 180 seconds.

- The experiments are completed when the fetal head is extracted from the vulva which corresponds to a distance from the ischial plan equals to $15 \mathrm{~cm}$. For this position the operators can remove their forceps and the fetus becomes a newborn.

These conditions correspond to the situation of a parturient considered as too tired to expel her fetus alone. Her IEF is not sufficient to expel the fetus and she is not able to produce VEF. Obstetricians have to thus extract the fetus with the forceps. During their first attempts, novices had no access to any information about the simulated maternal forces and applied their ITF whenever they wished. Then, they could visualize the simulated forces on a screen which allowed them to synchronize the different forces at the right moment. They thus carried out six forceps extractions: three attempts without synchronization and three with synchronization. Six novices are evaluated on the BirthSIM simulator.

\section{Synchronization concept}

Fig. 7 shows the ITF applied by one novice during attempts without synchronization. Similar plots are obtained with the other novices.

On this figure, the dotted line represents the maternal expulsive forces (IEF) and the plain lines correspond to the 


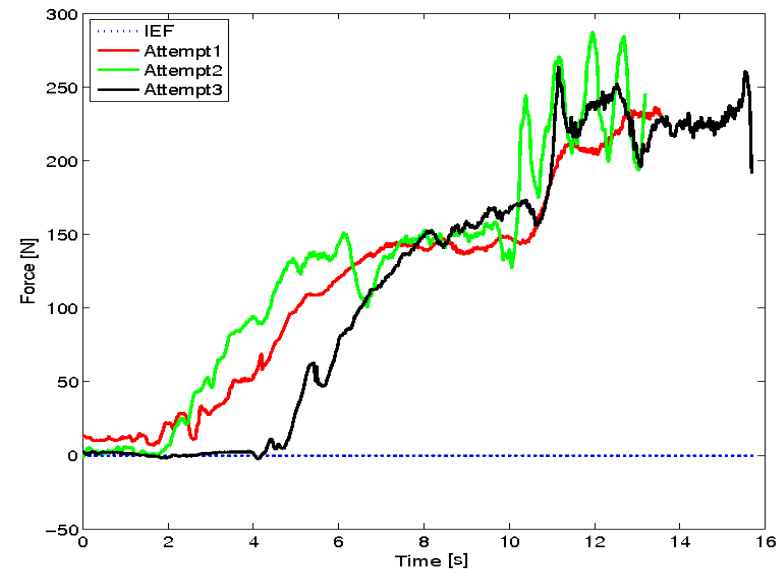

Figure 7. ITF applied by novice 1 without synchronization

ITF applied by the novice during the different attempts. As he had no information displayed on the screen, the novice did not wait for the IEF. In this situation, hiss ITF is thus the only expulsive force applied on the fetal head. Experimental data show that the ITF amplitude can reach up to 287 N. For his three attempts, the novice's ITF is beyond $200 \mathrm{~N}$ which is the upper recommended limit [24]. Similar results are obtained for the other novices.

Concerning the fetal head displacement due to the ITF applied without synchronization, it is represented on Fig. 8.

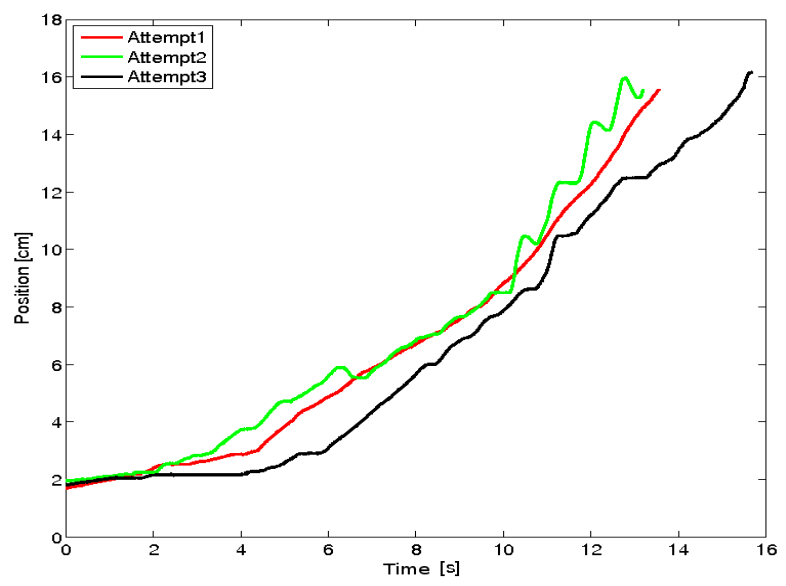

Figure 8. Fetal head displacement during attempts without synchronization

The displacement of the fetal head should be as linear as possible to avoid any back and forth displacements which could lead to dramatic consequences either for the mother or for the fetus. During attempts without synchronization it is difficult for novices to ensure a displacement as linear as possible.

Concerning the attempts with synchronization, the applied ITF are shown on Fig. 9. During these attempts, the IEF is displayed on a screen which allows the novices to wait for the appropriate moment before applying their ITF $(\approx 30$ seconds after the beginning of the experiment).

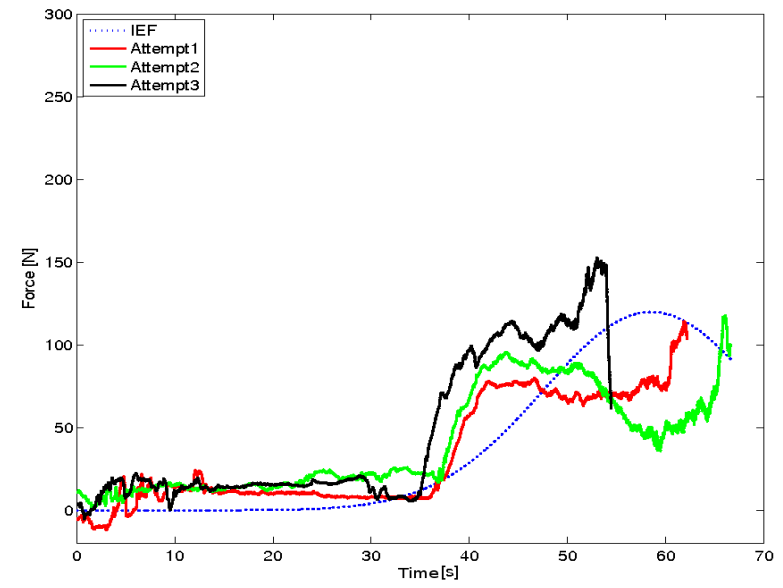

Figure 9. ITF applied by novice 1 with synchronization

The maximum ITF amplitude is approximately $150 \mathrm{~N}$. Concerning the fetal head displacement, it is represented on Fig. 10.

The conclusion of this experiment is while the applied ITF respects the concept of synchronization, the fetal head is not subjected to back and forth displacements and moves smoother. Similar results are obtained with the other novices, their numerical results are summed up in Tab. II and Tab. III. In order to complete the analysis, not only the ITF maximum amplitude is studied but also its behavior in space 


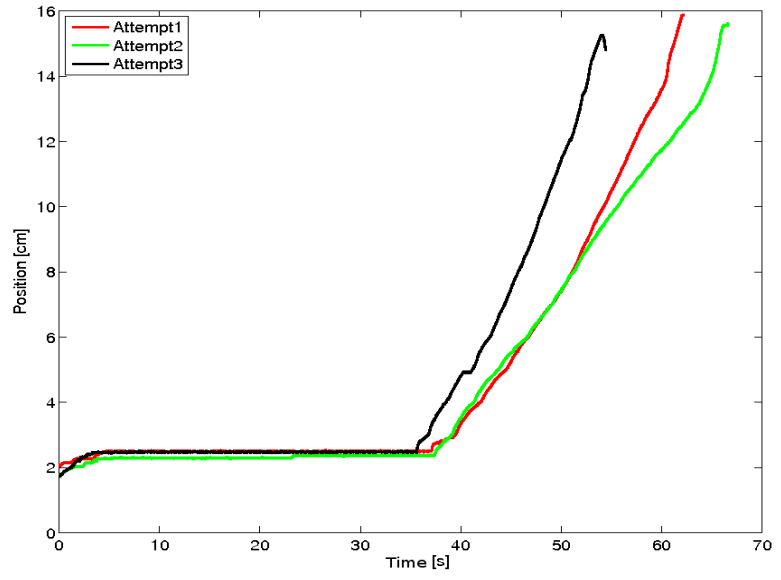

Figure 10. Fetal head displacement during attempts with synchronization

and in time, the ITF work and the ITF average are also computed. Tab. II gathers the numerical results of the attempts carried out without synchronization, whereas Tab. III gathers the numerical results of the attempts carried out with synchronization. The results correspond to the average of the different evaluation criteria over the three attempts. The values in brackets correspond to the standard deviation.

Table II

RESULTS OF THE ATTEMPTS CARRIED OUT WITHOUT THE SYNCHRONIZATION CONCEPT

\begin{tabular}{||c|c|c|c||}
\hline Evaluation & Average & Maximum & ITF \\
Criteria & ITF [N] & ITF [N] & work [J] \\
\hline Novice 1 & $119(5)$ & $262(25)$ & $29(6)$ \\
Novice 2 & $86(6)$ & $207(61)$ & $29(1)$ \\
Novice 3 & $82(28)$ & $156(54)$ & $25(5)$ \\
Novice 4 & $106(5)$ & $150(5)$ & $30(4)$ \\
Novice 5 & $107(24)$ & $207(32)$ & $23(7)$ \\
Novice 6 & $123(14)$ & $224(11)$ & $30(4)$ \\
\hline Average & $104(14)$ & $201(31)$ & $28(4)$ \\
\hline
\end{tabular}

As expected the ITF amplitude applied during the attempts with synchronization is smaller than the ITF amplitude obtained without synchronization. The evolution of the numerical
Table III

RESULTS OF THE ATTEMPTS CARRIED OUT WITH THE SYNCHRONIZATION CONCEPT

\begin{tabular}{||c|c|c|c||}
\hline Evaluation & Average & Maximum & ITF \\
Criteria & ITF [N] & ITF [N] & work [J] \\
\hline Novice 1 & $66(13)$ & $128(21)$ & $22(3)$ \\
Novice 2 & $79(34)$ & $185(63)$ & $21(6)$ \\
Novice 3 & $54(21)$ & $123(40)$ & $18(5)$ \\
Novice 4 & $50(15)$ & $102(17)$ & $17(3)$ \\
Novice 5 & $71(2)$ & $124(11)$ & $20(2)$ \\
Novice 6 & $63(17)$ & $102(13)$ & $23(4)$ \\
\hline Average & $64(17)$ & $127(27)$ & $20(4)$ \\
\hline
\end{tabular}

values obtained between the two experiments are summed up in Tab IV.

Table IV

DECREASE OF PARAMETER VALUES BETWEEN ATTEMPTS WITHOUT AND WITH SYNCHRONIZATION IN \%

\begin{tabular}{||c|c|c|c|}
\hline $\begin{array}{c}\text { Evaluation } \\
\text { Criteria }\end{array}$ & $\begin{array}{c}\text { Average } \\
\text { ITF [N] }\end{array}$ & $\begin{array}{c}\text { Maximum } \\
\text { ITF [N] }\end{array}$ & $\begin{array}{c}\text { ITF } \\
\text { work [J] }\end{array}$ \\
\hline Novice 1 & 44 & 51 & 26 \\
Novice 2 & 8 & 11 & 26 \\
Novice 3 & 34 & 21 & 30 \\
Novice 4 & 53 & 32 & 43 \\
Novice 5 & 34 & 40 & 10 \\
Novice 6 & 48 & 55 & 24 \\
\hline Average & 37 & 35 & 26 \\
\hline
\end{tabular}

By comparing the attempts with or without synchronization, we notice that for all novices, their values have decreased. We did not take into account the duration because the extraction lasted longer for the attempts with synchronization due to the fact that the operators have to wait before the IEF is triggered. As the aim of these experiments is to make novices aware of the involved forces and not to simulate an emergency 
procedure, the duration is not a determinant parameter.

\section{DISCUSSION}

In 1933, Wylie used a forceps, which was instrumented with a dynamometer, to measure the force applied during an extraction [9]. He demonstrated that the tractive force can reach $300 \mathrm{~N}$ during a forceps extraction. He also shown that the force depends on the parturient parity (number of children she had already given birth to) and the obstetrician subjectivity because they are not always aware of the force they applied while extracting a fetus.

Fleming et al. instrumented a forceps with strain gauges [10] and Pearse used it to demonstrate that the average maximal force is around $190 \mathrm{~N}$ [25]. In 1966, Kelly modified slightly the position of the strain gauges on the forceps: instead of being on the middle part of the forceps, they are now place on the extremity of the forceps which is in contact with the fetal head. In [11] he demonstrated that beyond $230 \mathrm{~N} \mathrm{50 \%}$ of the new borns have injuries. Nowadays the upper recommended limit of the extraction force is around $200 \mathrm{~N}$ [24].

The disparity of the literature results confirm the difficulty to carry out in vivo measurements. Indeed, it is difficult to measure the real tractive force applied on the fetal head due to the impossibility to equip the fetus with sensors and the uniqueness of each delivery. This difficulty leads researchers to help obstetrician novices to learn the correct force to apply outside the delivery room. Leslie et al. thus proposed a training using a mechanical arm on which forceps are attached and a force sensor is mounted [26]. In our case the interface is an anthropomorphic manikin, and forceps are not attached, which increase the immersion in the simulation. Our results lead to the same conclusion: novice obstetricians need training to be aware of the force they can exert and this training is not always obvious to be carried out in the delivery ward.

One major advantage of a simulator is not only to offer a realistic risk-free training, but also to enable the study of other parameters which are difficult to measure during a delivery such as the fetal head displacement and the real force applied on the head. A simulator can thus also be used as a research tool for obstetricians to compare and validate new techniques and instruments.

Concerning the force sensor used to measure the ITF applied by operators, it is only used as a measurement tool. We did not use it in a force control because we wanted first to check if we managed to obtain results close to the literature. Moreover the position feedback control used reproduces a realistic displacement of the fetal head which tends to be maintained inside the pelvis by the pelvic muscles and thus to have back and forth displacements.

\section{CONCLUSION}

The control law presented and implemented in the electropneumatic component of the BirthSIM simulator answers obstetrician needs. Different scenarios are available on the BirthSIM simulator. In this paper we mainly focus on the procedure which simulates a difficult forceps delivery. The control law is based on a position feedback control with a sliding gain $K_{x}$ which allows to modify the stiffness of the system and to reproduce the different forces involved during 
a delivery. The aim of the experiments in this paper was to demonstrate the interest of the force synchronization concepts to novices and to make them aware of the forces involved during a delivery.

Their extraction manipulations were then compared. This comparison leads to the conclusion that novices do not exert the same effort and need to practice before mastering the ITF they exert. None of the trained novices obtained the same results, they therefore need a personalized training to help them acquiring experience. This personalization is not possible in the delivery ward but can be carried out on a simulator. We also studied the fetal head displacement and showed to the novices the displacement due to the force they exert. If the head is subjected to back and forth displacements, this may cause dramatic injuries to the parturient and the fetus. This first experience acquired on a simulator will likely help novices feel more confident and to be ready when a real instrumental delivery occurs. Concerning the realistic approximation of maternal expulsive forces and instrumental tractive forces generated in these experiments, the values obtained during the delivery simulation are compared to the literature results, and are within their range.

A training program is actually under development to allow novices to improve their manipulations and to proceed to a risk-free instrumental delivery under the supervision of an expert. This new measurement campaign should lead to more representative results.

\section{REFERENCES}

[1] O. Dupuis, R. Silveira, T. Redarce, A. Dittmar, and R.-C. Rudigoz. Operative vaginal delivery rate and neonatal associated complications in 2002 in the AURORE hospital network. Gynécologie Obstétrique et Fertilité, 31(11):920-926, 2003.

[2] T. Sielhorst, T. Obst, R. Burgkart, R. Riener, and N. Navab. An augmented reality delivery simulator for medical training. In Augmented environments for Medical Imaging including Augmented Reality in Computer-aided Surgery (AMI ARCS'04), pages 11-20, Rennes, France, 2004.

[3] R. Lapeer, M. S. Chen, and J. Villagrana. Simulating obstetric forceps delivery in an augmented environment. In Augmented environments for Medical Imaging including Augmented Reality in Computer-aided Surgery (AMI ARCS'04), Rennes, France, 2004.

[4] Richard Moreau, Minh Tu Pham, Tanneguy Redarce, and Olivier Dupuis. A new learning method for obstetric gestures using the BirthSIM simulator. In IEEE International Conference on Robotics and Automation (ICRA'07), pages 2279-2284, Roma, Italy, 10-14 April 2007.

[5] R. Moreau, V. Ochoa, M.T. Pham, P. Boulanger, T. Redarce, and O. Dupuis. A method to evaluate skill transfer and acquisition of obstetric gestures based on the curvatures analysis of the position and the orientation. Elsevier, Journal of Biomedical Informatics, 41:991-1000, 2008. doi:10.1016/j.jbi.2008.03.012.

[6] R. Silveira, M. T. Pham, T. Redarce, M. Betemps, and O. Dupuis. A new mechanical birth simulator: BirthSIM. In IEEE/RSJ International Conference on Intelligent Robots and Systems, (IROS'04), volume 4, pages 3948-3953, 28 Sept.-2 Oct. 2004.

[7] E. Papiernik, D. Cabrol, and J.-C. Pons. Obstétrique. Flammarion Médecine-Sciences, 1997. ISBN 2257150457.

[8] J.-M. Thoulon. Le monitorage électronique foctal : la cardiotocographie. Masson, $2^{\text {ème }}$ edition, 1991. ISBN 2-225-82251-4.

[9] B. Wylie. Traction in forceps deliveries. American Journal of Obstetrics and Gynecology (AJOG), pages 425-433, 1933.

[10] A. R. Fleming, K. R. Brandeberry, and W. H. Pearse. Introduction of a metric forceps. American Journal of Obstetrics and Gynecology (AJOG), 78:125-133, 1959.

[11] J. V. Kelly and G. Sines. An assessment of the compression and 
traction forces of obstetrical forceps. American Journal of Obstetrics and Gynecology (AJOG), 96:521-537, 1966.

[12] A. Moolgoaker. A new design of obstetric forceps. Obstetrics and Gynecology, 69:450-457, 1962.

[13] P. Rosa. Defense of suction grip extraction. Brux Med, 35:1590-1597, 1955.

[14] R. Moreau, M.T. Pham, R. Silveira, T. Redarce, X. Brun, and O. Dupuis. Design of a new instrumented forceps: Application to safe obstetrical forceps blade placement. IEEE Transactions on Biomedical Engineering, 54(7):1280-1290, July 2007.

[15] American College of Obstetricians and Gynecologist (ACOG). Operative vaginal delivery. Technical report, ACOG Practice Bulletin No 17, June 2000.

[16] G. Cunningham, L. Gilstrap, K. Leveno, S. Bloom, J. Hauth, and K. Wenstrom. Williams Obstetrics. the McGraw-Hill Companies, $22^{\text {nd }}$ edition, 2005. ISBN 0071413154.

[17] O. Olaby, R. Moreau, X. Brun, O. Dupuis, and T. Redarce. Automatic childbirth procedures implanted on the BirthSIM simulator. In IEEE/RSJ International Conference on Intelligent Robots and Systems (IROS'06), pages 2370-2375, Beijing, China, 2006.

[18] B. L. Andersen. The Analysis and Design of Pneumatic Systems. Wiley, New-York, 1967.

[19] J. L. Shearer. Study of pneumatic processes in the continuous control of motion with compressed air. parts I and II. Trans. Am. Soc. Mech. Eng., 78:233-249, 1956.

[20] X. Brun, D. Thomasset, and E. Bideaux. Influence of the process design on the control strategy: application in electropneumatic field. Control Engineering Practice, 10(7):727-735, July 2002.

[21] X. Brun, S. Sesmat, D. Thomasset, and Scavarda S. A comparative study between two control laws of an electropneumatic actuator. In European Control Conference (ECC'99), pages F1000-5, Karlsruhe, Germany, Sept. 1999.

[22] O. Olaby, S. Sesmat, X. Brun, T. Redarce, and E. Bideaux. Characterization and modelling of a FESTO proportionnal valve in aim of control synthesis. In The $6^{\text {th }}$ JFPS International Symposium on Fluid Power, Tsubaka, Japan, November 7-10 2005.

[23] X. Shen and M. Goldfarb. Simultaneous force and stiffness control of a pneumatic actuator. Journal of Dynamic Systems, 129:425-434, 2007.
[24] P.C. Dennen. Dennen's Forceps Deliveries. F.A. Davis, $3^{\text {rd }}$ ed. edition, 1989. ISBN: 0803625111.

[25] W. H. Pearse. Electronic recording of forceps delivery. American Journal of Obstetrics and Gynecology (AJOG), 86:43-51, 1963.

[26] K. Leslie, P. Dipasquale-Lehnerz, and M. Smith. Obstetrics forceps training using visual feedback and the isometric strength testing unit. Obstetrics \& Gynecology, 105:377:382, 2005. 